\title{
Bulk PPKTP by crystal growth from high temperature solution
}

\author{
A. Peña ${ }^{\text {a }}$, B. Ménaert ${ }^{\text {a }}$, B. Boulanger ${ }^{\text {a }}$, F. Laurell ${ }^{\text {b }}$, C. Canalias ${ }^{\text {b }}$, V. Pasiskevicius ${ }^{\text {b }}$, L. \\ Ortega ${ }^{a}$, P. Segonds ${ }^{\text {a }}$, J. Debray ${ }^{\mathrm{a}}$, C. Félix ${ }^{\mathrm{a}}$, \\ ${ }^{a}$ Institut Néel CNRS/UJF, 25 rue des Martyrs, BP 166, F38042 Grenoble Cedex 9, France \\ ${ }^{b}$ Laser Physics, Applied Physics Department, Royal Institut of Technology, Roslagstullsbacken 21, 10691 Stockholm, Sweden
}

\begin{abstract}
Periodically-poled ferroelectric crystals show unprecedented efficiency and properties otherwise impossible to reach. Unfortunately, the sample thickness obtainable today limits their use to low and moderate power application. With the aim of increasing the size of periodically domain-structured crystals with a controlled and regular grating period, we proposed an epitaxial growth process using seeds made of thin plates domain engineered by electric field poling. We demonstrated this process with the ferroelectric crystal $\mathrm{KTiOPO}_{4}(\mathrm{KTP})$ which is one of the most promising candidate materials for that purpose. The poling step requires a sample exhibiting $(001)$ and $(00 \overline{1})$ faces, so that the growth step has to be performed onto these faces. This constraint is a difficulty to circumvent as these faces are not present in the standard equilibrium morphology. It is then necessary to find the growth conditions enabling to work below the roughening temperature of these faces. By using a high temperature solution method, the so-called "flux method", and by choosing an appropriate chemical composition of the flux solution, we obtained periodically domain-structured KTP layers with thicknesses up to $800 \mu \mathrm{m}$ and regular periodicity onto $(001)$ and $(00 \overline{1})$ faces of the initial PPKTP seeds.
\end{abstract}

Keywords: Epitaxy, Microstructures, Nonlinear Optics

\section{Introduction}

Periodic inversion of ferroelectric domains in the nonlinear crystal $\mathrm{KTiOPO}_{4}$ (KTP) [1-4] has been extensively used in order to achieve frequency conversion by Quasi-Phase-Matching (QPM) [5], which is more efficient than by performing Birefringence-PhaseMatching (BPM) in a single domain KTP crystal. Until now, commercially Periodically Poled KTP (PPKTP) crystals were fabricated by the electric field poling technique, based on the inversion of the spontaneous polarization $P_{s}$ by applying a high electric field to a single domain crystal [6]. Such samples are used in low energy devices [7,8] because of their small thickness along $P_{s}$ direction.

In situ growth techniques had been then proposed in order to obtain larger size PPKTP samples from single domain KTP crystals [9]. But unfortunately the regularity of the obtained grating period was not sufficient for a use in practical Second Harmonic Generators (SHG) or Optical Parametric Oscillators (OPO). The same limitation had occurred when trying in situ growth of periodically-poled $\mathrm{LiNbO}_{3}$ (PPLN) crystal, another ferroelectric medium widely used for QPM [10]. In situ growth techniques had also been used in patterned nonlinear crystals in order to achieve frequency conversion by QPM: 1-mm-thick Orientation-Patterned GaAs (OP-GaAs) have been recently obtained by Low-pressure hydride vapor phase epitaxy (HVPE) [11] enabling the generation of a mid-IR continuum by optical parametric generation (OPG) [12].

Here we propose a crystal growth process starting from a thin seed of PPKTP obtained in a previous step from electric field poling, with the objective to obtain thicker size samples. We consider a high temperature solution growth method enabling to perform the crystal growth below the Curie temperature of the crystal and below the roughening temperature of the relevant crystallographic faces, i.e. the two faces (001) and $(00 \overline{1})$ that are perpendicular to $P_{s}$, so that a thick PPKTP crystal with the same grating periodicity than the one of the seed can be obtained.

\section{Definition of the growth conditions}

The growth process should be done in the temperature range of the ferroelectric phase of KTP, i.e. below its Curie temperature [13], and also below the roughening temperature of $(001)$ and $(00 \overline{1})$ faces [14]. The first condition is necessary to maintain the periodic domain structure of the initial seed during the growth process. The second condition, which is imposed by the geometry of the thin PPKTP seeds that exhibit large artificial (001) and $(00 \overline{1})$ faces, allows us to suppress undesirable capping process that would lead to a reconstruction of the crystal with a pyramidal domain shape formed by $\{201\}$ and $\{011\}$ faces [15]. 
As shown in fig. 1, the standard flux used to grow KTP, i.e. $\mathrm{K}_{6} \mathrm{P}_{4} \mathrm{O}_{13}$, is not suitable for that purpose because its lower growth limit is $1098 \mathrm{~K}[16,17]$, which is over the roughening temperature of $(001)$ and $(00 \overline{1})$ faces. Below this temperature, the crystals that are obtained belong to a polyphosphate complex phase [18]. Here we propose to use a flux containing a mixture of $\mathrm{KPO}_{3}$ and $\mathrm{KF}$ from which KTP can be obtained at temperatures as low as 873 $\mathrm{K}$, corresponding to the eutectic point of the system [19]. The increasing of the temperature range compared with that of the standard flux $\mathrm{K}_{6} \mathrm{P}_{4} \mathrm{O}_{13}$ is due to a higher ionic character of the flux, thanks to the presence of $\mathrm{KF}$ preventing undesired polyphosphate complex phases.

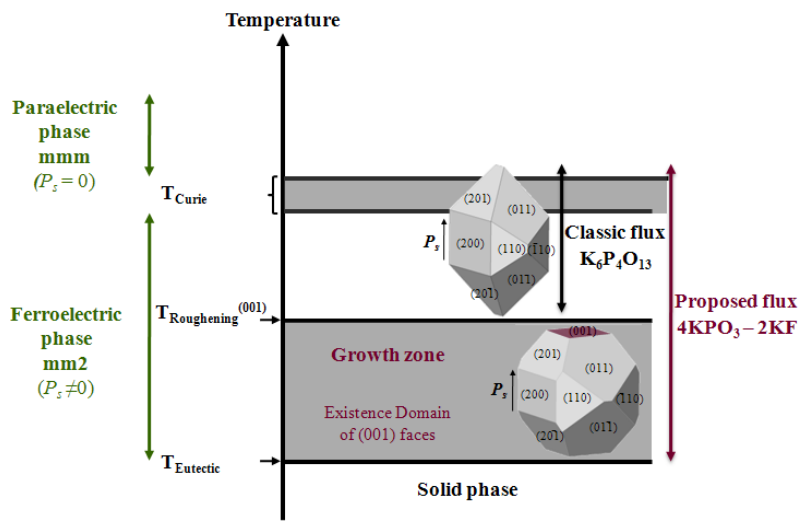

Fig 1. Schematic thermal range of $\mathrm{KTiOPO}_{4}(\mathrm{KTP})$ ferroelectric and paraelectric phases and possible equilibrium morphologies in the paraelectric phase using two different fluxes.

\section{Crystal growth results}

The growth experiments were performed in $4 \mathrm{KPO}_{3}-2 \mathrm{KF}$ flux, which allowed us to keep the same molar ratio as in the case of $\mathrm{K}_{6} \mathrm{P}_{4} \mathrm{O}_{13}$, i.e. $\mathrm{K} / \mathrm{P}=1.5$, so that it was possible to use experimental data previously published in the case of $\mathrm{K}_{6} \mathrm{P}_{4} \mathrm{O}_{13}$ [20]. The corresponding reaction constant $(\mathrm{k})$ as a function of temperature was deduced by using an ionic solubility model [21,22], which led to the determination of the molar enthalpy of crystallization, i.e. $\Delta H=6001.5 \mathrm{cal} / \mathrm{mol}$, and then to the calculation of the equilibrium curve of fig. 2 . We validated these calculations by the thermo-gravimetric measurement of the saturation temperature corresponding to the molar composition $0.103 \mathrm{KTP}-0.598 \mathrm{KPO}_{3}-0.299 \mathrm{KF}$ : we found $1005.5 \pm 0.1 \mathrm{~K}$, which is very close to the theoretical value, i.e. $1003 \mathrm{~K}$.

Preliminary growth experiments were realized in order to check whether $c$ and $\bar{c}$ faces appear close to the calculated roughening temperature value, $T_{R}=1053 \mathrm{~K}$, predicted by Bennema [14] from the Rijpkema model [23]. The external morphology of small crystals grown by spontaneous nucleation in $4 \mathrm{KPO}_{3}-2 \mathrm{KF}$ flux with different initial molar composition was visualized by an optical microscopy and a scanning electron microscope (SEM). The saturation temperatures were predicted using the equilibrium curve shown in fig. 2. The crystals exhibited $(001)$ and $(00 \overline{1})$ faces below $1048 \mathrm{~K}$ as shown in fig. 3. So this temperature is close to the calculated one, i.e. $1053 \mathrm{~K}$ [14]. Note that these two faces can also be stabilized over their roughening temperature by using a $\mathrm{K}_{6} \mathrm{P}_{4} \mathrm{O}_{13}-\mathrm{PbO}$ flux [24].

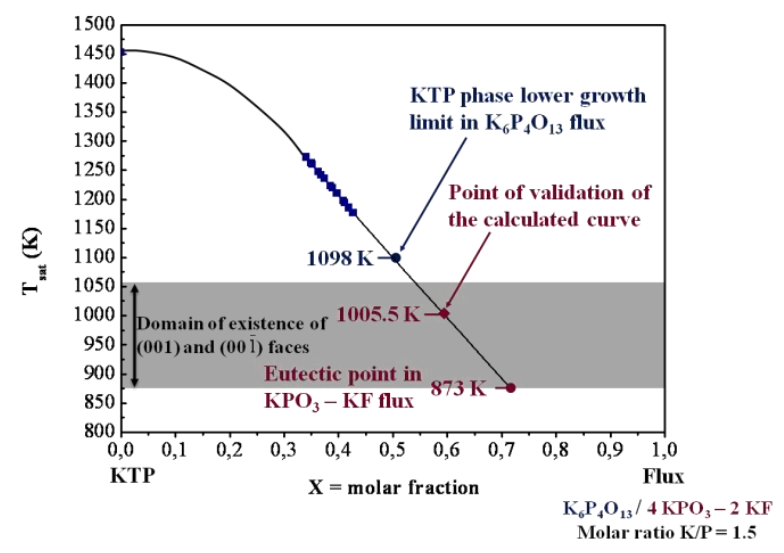

Fig 2. $\mathrm{KTiOPO}_{4}(\mathrm{KTP})$ pseudo binary phase diagram using $\mathrm{K}_{6} \mathrm{P}_{4} \mathrm{O}_{13}$ and $4 \mathrm{KPO}_{3}-2 \mathrm{KF}$ flux. Parameter $\mathrm{X}$ is the molar fraction, i.e. $\frac{\left[K_{6} P_{4} O_{13}\right]}{[K T P]+\left[K_{6} P_{4} O_{13}\right]}$ or $\frac{\left[4 K P O_{3}: 2 K F\right]}{[K T P]+\left[4 K P O_{3}: 2 K F\right]} . \mathrm{T}_{\text {sat }}$ is the saturation temperature of KTP.

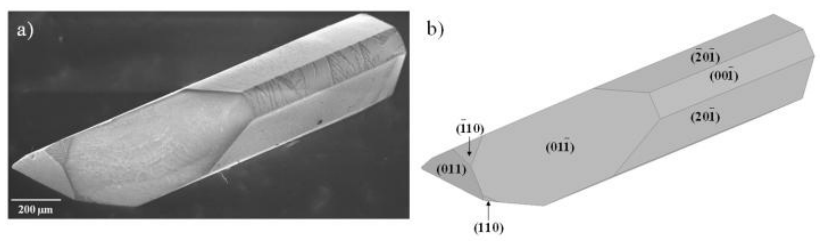

Fig 3. a) SEM image of a single KTP crystal grown by spontaneous nucleation in a $0.120 \mathrm{KTP}-0.586 \mathrm{KPO}_{3}-0.293$ $\mathrm{KF}$ flux. b) Morphological representation of the grown crystal obtained with SHAPE program.

The best growth conditions to obtain good quality PPKTP layers grown onto (001) faces were determined by preliminary experiments using single domain KTP seeds. The epitaxial growth experiments were done in a solution with the same molar composition than the one used to validate the calculations done to obtain the equilibrium curve of fig. 2, i.e.: $0.103 \mathrm{KTP}-0.598 \mathrm{KPO}_{3}-0.299 \mathrm{KF}$. The saturation temperature of this solution is under the roughening temperature of $(001)$ and $(00 \overline{1})$, so that no capping process will be expected [15]. Good quality KTP layers of few hundred $\mu \mathrm{m}$ thickness were obtained by applying an over-saturation of around $2 \%$ and by alternatively rotate the initial seed with an angular speed of around $30 \mathrm{rpm}$.

These growth conditions were then used to grow thick PPKTP layers onto PPKTP seeds previously obtained by electric field poling, with a periodicity $\Lambda=38.86 \mu \mathrm{m}$. The results were very satisfying, with a propagation of the domains along the thickness of the as grown layer, as it shown in fig $4 \mathrm{~b}$. PPKTP layers of up to $800 \mu \mathrm{m}$ onto $(001)$ and $(00 \overline{1})$ faces were grown by using a growth ramp of $0.05 \mathrm{~K} / \mathrm{h}$ (fig. 4a). This cooling ramp, starting from an initial temperature of $1005.5 \mathrm{~K}$, allows us to have a constant matter transfer from the solution to the grown crystal during all the growth process. 
a)
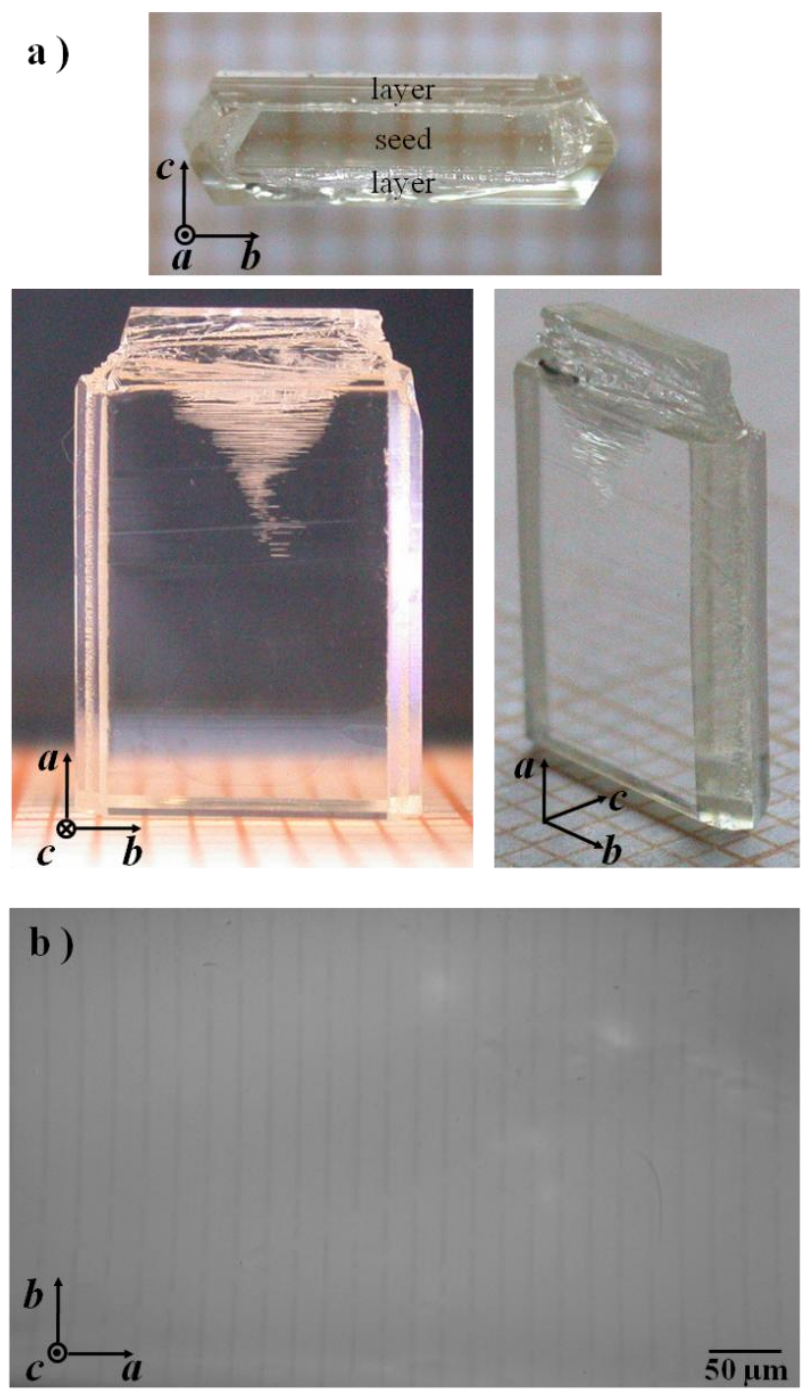

Fig 4. a) Three pictures of a single PPKTP crystal grown by liquid phase epitaxy in a vertical dipping configuration. The growth was performed from a solution with the molar composition $0.120 \mathrm{KTP}-0.586 \mathrm{KPO}_{3}-0.293 \mathrm{KF}$ and the initial seed used has a period grating $(\Lambda)$ equal to $38.86 \mu \mathrm{m}$. b) $\mathrm{SEM}$ image, at $200 \mu \mathrm{m}$ from the seed surface, of the $\mathbf{c}$ face of the grown layer.

\section{Characterizations}

A Scanning Electron Microscope (SEM) have been used to determine the quality of the interface between the seed and the growing layer, to visualize the propagation of the ferroelectric domains from the seed to the layer and also to determine the growth rate along $c$ direction. The interface is of very good quality and the domains propagate without any measurable variation in size from the seed surface till the layer surface [25]. Due to the high quality of the interface and to the fact that we used a homoepitaxial growth, it was not possible to see any contrast between seed and grown layer, with beam energies higher than $3 \mathrm{kV}$ and work distances higher than $6 \mathrm{~mm}$. The growth rate along $\mathbf{c}$ was determined by SEM and also by a gravimetric method, which provides the change in apparent weight during all the growth process. By both methods, we obtained a growth rate of $2 \mu \mathrm{m} / \mathrm{h}$.
The crystal quality was determined by X-ray diffraction from a $\theta-2 \theta$ scan as shown in fig 5. The full width at half maximum (FWHM) of the measured (004) reflection is equal to $0.006^{\circ}$ for both the substrate and growth layer, attesting of an equivalent quality. The difference between the diffraction peak angles, i.e. $2 \theta=33.859^{\circ}$ for the seed and $2 \theta=33.8599^{\circ}$ for the layer, corresponds to a mismatch $\Delta c=1 \times 10^{-3}$. This cell parameter variation is not surprising since the seed and the layer have not been grown from the same flux. There is also a difference of peak intensity between the seed and the layer, which comes from the difference of irradiated matter volumes due to the particular cut geometry of the sample as shown on the insert of fig. 5 .

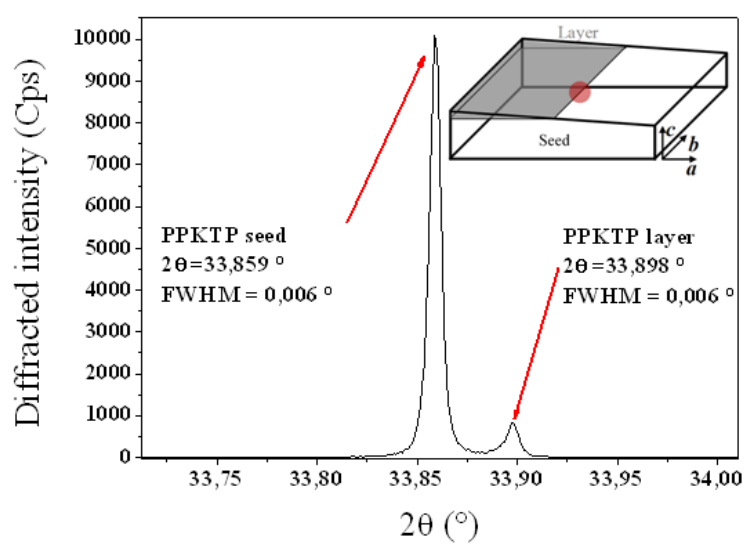

Fig 5. Diffraction $\theta-2 \theta$ scan for the (004) reflection of $\mathrm{KTiOPO}_{4}$ (KTP). Scheme of the cut edge PPKP sample (insert) in which the $\theta-2 \theta$ scan was recorded.

The grating period regularity of the ferroelectric domains in the grown layer as well as the replication of the initial periodically poled zone present in the initial seed had been checked by performing first order Quasi Phase Matching (QPM) Second Harmonic Generation (SHG) measurements. The obtained result [25] shows the perfect replica of the domain structure over the first 200 $\mu \mathrm{m}$ along $\mathbf{c}$ direction of the grown layer. $\mathrm{Up}$ to this clean area and till to the top of the layer $(800 \mu \mathrm{m})$, there was a strong diffusion of the laser source due to growth defects that are not connected with the propagation of the domains. New experiments are in progress in order to suppress these defects.

\section{Summary}

In summary, growth conditions to obtain thick PPKTP layers from PPKTP seeds have been determined. Up to now PPKTP layers with a thickness of $800 \mu \mathrm{m}$ along the $\boldsymbol{c}$ direction, onto (001) and $(00 \overline{1})$ faces of the initial PPKTP seed, have been obtained by epitaxial growth from a KTP$\mathrm{KPO}_{3}-\mathrm{KF}$ solution at temperatures lowers than $1005.5 \mathrm{~K}$. Crystalline quality as well as the domain grating periodicity of the grown layers are the same than in the seeds. We think that the proposed technology is well adapted to obtain PPKTP crystals as well as other periodically poled materials requiring the flux method. 


\section{Acknowledgements}

A. Peña acknowledges CNRS and AGAUR (2008 BP A) for the financial supports of her postdoctoral positions. The KTH group acknowledges support from the Linnaeus centre ADOPT, Swedish Research council (Vetenskapsrådet) and the Knut and Alice Wallenberg Foundation. All the authors wish to acknowledge Cristal Laser S.A. for providing single domain KTP crystals for the preliminary epitaxial growth experiments.

\section{References}

[1] H. Karlsson, F. Laurell, Appl. Phys. Lett. 71 (1997) 3474.

[2] M.C. Gupta, W.P. Risk, Alan C.G. Nutt, S.D. Lau, Appl. Phys. Lett. 63 (1993) 4136.

[3] W.P. Risk, S.D. Lau, Appl. Phys. Lett. 69 (1996) 3999.

[4] C. Canalias, V. Pasiskevicius, R. Clemens, F. Laurell, Appl. Phys. Lett. 82 (2003) 4233.

[5] M.M. Fejer, G.A. Magel, D. H. Jundt, R. L. Byer, IEEE J. Quantum Electron. 28 (1992) 2631.

[6] http://www.raicol.com/product_details.asp

[7] J.P. Fève, O. Pacaud, B. Boulanger, B. Ménaert, J. Hellström, V. Pasiskevicius, F. Laurell, Opt. Lett. 26 (2001) 1882.

[8] G.M. Gibson, M. Ebrahimzadeh, M.J. Padgett, M.H. Dunn, Opt. Lett. 24 (1999) 397.

[9] M. Roth, N. Angert, M. Tseitlin, J. Mater. Sci-Mater. Electron. 12 (2001) 429.

[10] V. Bermúdez, D. Callejo, E. Diéguez, J. Cryst. Growth 207 (1999) 303.

[11] C. Lynch, D.F. Bliss, T. Zens, A. Lin, J.S. Harris, P.S. Kuo, M.M. Fejer, J. Cryst. Growth 310 (2008) 5241.

[12] P.S. Kuo, K.L. Vodopyanov, M.M. Fejer, D.M. Simanovskii, X. Yu, J.S. Harris, D. Bliss, D. Weyburne, Opt. Lett. 31 (2006) 71.

[13] V.K. Yanovskii, V.I. Voronkova, Phys. Status Solidi A 93 (1986) 665.

[14] R.J. Bolt, P. Bennema, J. Cryst. Growth 102 (1990) 329.

[15] P.J. Halfpenny, L. O’Neill, J.N. Sherwood, G.s. Simpson, A. Yokotani, A. Miyamoto, T. Sasaki, S. Nakai, J. Cryst. Growth 113 (1991) 722.

[16] R.J. Bolt, M.H. van der Mooren, H. de Haas, J. Cryst. Growth 114 (1991) 141.

[17] J.C. Jacco, G.M. Loiacono, M. Jaso, G. Mizell, B. Greenberg, J. Cryst. Growth 70 (1984) 484.

[18] G.M. Loiacono, T.F. McGee, G. Kostecky, J. Cryst. Growth 104 (1990) 389.

[19] G. Marnier, U.S. patent (1990) 4961819.

[20] P.F. Bordui, J.C. Jacco, G.M. Loiacono, R.A. Stolzenberger, J. Cryst. Growth 84 (1987) 403.

[21] M. Temkin, Acta Phys. Chim. URSS 20 (1945) 411.

[22] W. van Erk, J. Cryst. Growth 46 (1979) 539.

[23] J.J.M. Rijpkema, H.J.F. Knops, P. Bennema, J.P. van der Eerden, J. Cryst. Growth 70 (1984) 484.

[24] M. Tseitlin, E. Mojaev, M. Roth, J. Cryst. Growth 312 (2010) 1055. [25] A. Peña, B. Ménaert, B. Boulanger, F. Laurell, C. Canalias, V. Pasiskevicius, P. Segonds, C. Félix, J. Debray, S. Pairis, Invited paper, Opt. Mater. Express 1 (2011) 185. 